\title{
Commentary Moving beyond tight glucose control to safe effective glucose control
}

\author{
James S Krinsley ${ }^{1}$ and Jean-Charles Preiser ${ }^{2}$
}

1Division of Critical Care, Stamford Hospital, 190 West Broad Street, Stamford, CT 06902, USA

${ }^{2}$ Department of General Intensive Care, University Hospital Centre of Liege, Domaine Universitaire de Liège, 4000 Liege, Belgium

Corresponding author: Jean-Charles Preiser, Jean-Charles.Preiser@chu.ulg.ac.be

Published: 16 May 2008

Critical Care 2008, 12:149 (doi:10.1186/cc6889)

This article is online at http://ccforum.com/content/12/3/149

(c) 2008 BioMed Central Ltd

\begin{abstract}
The impressive benefits related to the use of tight glucose control by intensive insulin therapy have not been reproduced until now in multicenter large-scale prospective randomized trials. Although the reasons for these failures are not entirely clear, we suggest the use of a stepwise approach - Safe, Effective Glucose Control - that will essentially target an intermediate blood glucose level. As compared with genuine tight glucose control, Safe, Effective Glucose Control - already used in many intensive care units worldwide - is intended to decrease the rate of hypoglycemia and the workload, while reducing the adverse effects of severe hyperglycemia.
\end{abstract}

In 2001, following in the path of the glycemic pioneers [1-3], the Leuven 1 investigators published their landmark study of intensive insulin therapy in a population of surgical intensive care unit (ICU) patients, targeting 80 to $110 \mathrm{mg} / \mathrm{dl}$ in the interventional arm [4]. This prospective, controlled randomized trial spurred clinicians in ICUs around the world to adopt tight glycemic control (TGC) [5]. Confirmation of the mortality benefit of TGC in a mixed medical-surgical ICU setting was seen in the nonrandomized Stamford study published nearly 3 years later $[6,7]$. An additional 2 years elapsed until the publication of the Leuven II study, performed in a medical ICU, which demonstrated reduced mortality in the predefined population of patients staying in the ICU for at least 3 days but not in the entire population [8]. Two subsequent multicenter studies - GLUCONTROL and VISEP - were terminated prematurely, mainly because of the occurrence of severe hypoglycemia without concurrent improvement in survival $[9,10]$.

Why were the benefits of TGC apparent in the earlier studies not confirmed in the more recently published work?

TGC demands a complex application of monitoring and dynamic treatment throughout the course of the patient's ICU stay; deficiencies in any number of institutional factors may doom the intervention to failure. Protocol-driven care is central to TGC, with frequent assessment of glycemic levels and responding adjustments in the administered treatment. The experience and skill of the nursing staff in the use of protocols will materially affect the probability that the treatment goals of the protocol are achieved. Moreover, the structural and organizational characteristics of the ICU may have a strong impact, especially in view of the high work burden imposed by TGC - estimated to consume up to 2 hours out of a 24-hour working day for the ICU nurse [11,12].

Appropriate data outcome tools greatly increase the chance that TGC will be practiced successfully. Glycemic reporting tools allow clinicians to know whether glycemic targets are being reached and, importantly, whether there is a significant rate of treatment-associated hypoglycemia. Ideally an ICU should also have an outcomes reporting tool - the ability to provide information such as severity-adjusted mortality and length of stay, complications and resource utilization. Positive feedback imparts a strong incentive to continue the effort needed to maintain effective implementation of TGC $[13,14]$.

One important difference between the early and later trials of TGC is the rate of treatment-associated severe hypoglycemia, defined as $<40 \mathrm{mg} / \mathrm{dl}$, found recently to confer increased risk of mortality in a large cohort of mixed medical-surgical ICU patients [15]. The Leuven I study reported an increase in the number of patients with severe hypoglycemia from $0.8 \%$ to $5.1 \%$, with no associated adverse consequences [4]. There was no increase in severe hypoglycemia in the Stamford study [6]. In contrast, the percentages of patients in the corresponding groups of the Leuven II study were $3.1 \%$ and $18.7 \%$ (25.1\% among patients in the ICU for longer than 5 days) [8]. In this study the occurrence of severe hypoglycemia was independently associated with mortality on multivariable analysis and resulted in an attenuation of the survival benefit of TGC [8].

$\mathrm{ICU}=$ intensive care unit; SEGC = Safe, Effective Glycemic Control; TGC = tight glycemic control. 
Similarly, the percentage of patients sustaining severe hypoglycemia among patients in the interventional arm versus the control arm of the GLUCONTROL trial was 2.7\% versus $9.8 \%$ [9]; the corresponding rates in the VISEP trial were $4.1 \%$ and $17.0 \%$ [10]. It is possible that differences in monitoring technology and testing frequency may explain some of the differences in the rates of severe hypoglycemia when comparing the Leuven 1 study, which exclusively used arterial blood from indwelling arterial catheters, with the later studies. A growing literature has described the limitations of capillary glucose measurement in the critically ill patient, especially in the lower ranges targeted by these trials $[16,17]$.

It is likely that an additional factor - glycemic variability - has played a role in explaining the divergent outcomes of these different interventional trials $[18,19]$. A new evaluation of glycemic variability, defined as the standard deviation of the mean glucose level during the ICU stay, suggests that glycemic variability may be an even more important predictor of mortality in the critically ill patient than is the mean glucose level [20]. It is intriguing to note that while the mean (standard deviation) morning glucose levels of the control and interventional arms of the Leuven I study were 153 (33) mg/dl versus 103 (19) mg/dl [4], the corresponding results for the Leuven II study were 153 (31) mg/dl versus 111 (29) mg/dl [8]. Glycemic control improved in the second study but, perhaps, glycemic variability was unchanged.

We are forced, ultimately, to conclude that the Leuven I study may have set the bar too high: TGC, with a glycemic target of 80 to $110 \mathrm{mg} / \mathrm{dl}$ is, simply, too tight to practice safely and effectively. If TGC cannot be implemented safely and effectively in a research setting leading to a published interventional trial, then it probably cannot be implemented safely and effectively by most ICU teams.

Instead of TGC, we propose a stepwise approach defining a new standard - Safe, Effective Glycemic Control (SEGC) $[21,22]$. SEGC involves, first, adoption of a safe glycemic target appropriate to the skills, experience and available tools of the ICU that does not result in a significant increase in the rate of hypoglycemia. A glycemic target of 80 to $150 \mathrm{mg} / \mathrm{dl}$ is not unreasonable for an ICU to choose initially; implementation can subsequently lead to downward revision of the glycemic goal. Effective implementation of TGC involves successful attainment of glycemic goals with minimum variability. The use of appropriate data monitoring tools, for both glycemic results and relevant clinical outcomes, is essential for SEGC. Finally, sensible utilization of existing monitoring technologies is mandatory for SEGC.

Preliminary clinical evaluations of the accuracy of continuous or near-continuous glucose monitors have been published recently [23-25]. These devices offer the promise of a reduction in severe hypoglycemia, glycemic variability and the nursing work burden, and will probably become a corner- stone of SEGC. The goals of the SEGC mandate team collaboration are to create and apply glycemic protocols, and the appropriate use of all of the data and monitoring tools that we currently have in our armamentarium, as well as rapid employment of new tools as they are developed. Our patients deserve no less.

\section{Competing interests}

The authors declare that they have no competing interests.

\section{References}

1. Malmberg K, Ryden L, Efendic S, Herlitz J, Nicol P, Waldenström A, Wedel $\mathrm{H}$, Welin L: Randomized trial of insulin-glucose infusion followed by subcutaneous insulin treatment in diabetic patients with acute myocardial infarction: effects on mortality at 1 year. J Am Coll Cardiol 1995, 26:57-65.

2. Furnary AP, Gao G, Grunkemeier GL, Wu Y, Zerr KJ, Bookin SO, Floten HS, Starr A: Continuous insulin infusion reduces mortality in patients with diabetes undergoing coronary artery bypass grafting. J Thorac Cardiovasc Surg 2003, 125:10071021.

3. Lazar HL, Chipkin SR, Fitzgerald CA, Bao Y, Cabral H, Apstein CS: Tight glycemic control in diabetic coronary artery bypass graft patients improves perioperative outcomes and decreases recurrent ischemic events. Circulation 2004, 109: 1497-1502.

4. Van den Berghe G, Wouters P, Weekers F, Verwaest C, Bruyninckx F, Schetz M, Vlasselaers D, Ferdinande P, Lauwers P, Bouillon R: Intensive insulin therapy in the critically ill patients. N Engl J Med 2001, 345:1359-1367.

5. Wilson M, Weinreb J, Hoo GW: Intensive insulin therapy in critical care: a review of 12 protocols. Diabetes Care 2007, 30: 1005-1011.

6. Krinsley JS: Effect of an intensive glucose management protocol on the mortality of critically ill adult patients. Mayo Clinic Proc 2004, 79:992-1000.

7. Krinsley JS: Glycemic control, diabetic status, and mortality in a heterogeneous population of critically ill patients before and during the era of tight glycemic management: six and onehalf years experience at a university-affiliated community hospital. Semin Thorac Cardiovasc Surg 2006, 18:317-325.

8. Van den Berghe G, Wilmer A, Hermans G, Meersseman W, Wouters PJ, Milants I, Van Wijngaerden E, Bobbaerts H, Bouillon $\mathrm{R}$ : Intensive insulin therapy in the medical ICU. $N$ Engl J Med 2006, 354:449-461.

9. Devos P, Preiser JC, Mélot C, on behalf of the Glucontrol Steering Committee: Impact of tight glucose control by intensive insulin therapy on ICU mortality and the rate of hypoglycaemia: final results of the Glucontrol study [abstract]. Intensive Care Med 2007, 33:S189.

10. Brunkhorst FM, Engel C, Bloos F, Meier-Hellmann A, Ragaller M, Weiler N, Moerer O, Gruendling M, Oppert M, Grond S, Olthoff D, Jaschinski U, John S, Rossaint R, Welte T, Schaefer M, Kern P, Kuhnt E, Kiehntopf M, Hartog C, Natanson C, Loeffler M, Reinhart K, German Competence Network Sepsis (SepNet): Intensive insulin therapy and pentastarch resuscitation in severe sepsis. N Engl J Med 2008, 358:125-139.

11. Aragon D: Evaluation of nursing work effort and perceptions about blood glucose testing in tight glycemic control. $\mathrm{Am} J$ Crit Care 2006, 15:370-377.

12. Perreaux J, Devos P, Preiser JC, Melot C, on behalf of the Glucontrol Steering Committee: Impact of the implementation of tight glucose control by intensive insulin therapy on the nursing workload data from the Glucontrol study [abstract]. Intensive Care Med 2007, 33(Suppl 2):S54.

13. Gerard SO, Neary V, Apuzzo D, Giles ME, Krinsley J: Implementing an intensive glucose management initiative: strategies for success. Crit Care Nurs Clin North Am 2006, 18:531-543.

14. Krinsley JS: Translating evidence into practice in managing impatient hyperglycemia. J Hosp Med 2007, 2(Suppl 1):13-19.

15. Krinsley JS, Grover A: Severe hypoglycemia in critically ill patients: risk factors and outcomes. Crit Care Med 2007, 35: 2262-2267. 
16. Kanji S, Buffie J, Hutton B, Bunting PS, Singh A, McDonald K, Fergusson D, McIntyre LA, Hebert PC: Reliability of point-ofcare testing for glucose measurement in critically ill adults. Crit Care Med 2005, 33:2778-2885.

17. Mann EA, Salinas J, Pidcoke HF, Wolf SE, Holcomb JB, Wade $\mathrm{CE}$ : Error rates resulting from anemia can be corrected in multiple commonly used point-of-care glucometers. J Trauma 2008, 64:15-20.

18. Egi M, Bellomo R, Stachowski E, French CJ, Hart G: Variability of blood glucose concentrations and short-term mortality in critically ill patients. Anesthesiology 2006, 105:244-252.

19. Ali N, O'Brien J, Dungan K, Phillips G, Marsh C, Lemeshow S, Connors A, Preiser JC: Glucose variability is independently associated with mortality in patients with sepsis [abstract]. Crit Care Med 2007, 35:A924.

20. Krinsley JS: Glycemic variability: a strong, independent predictor of mortality in critically ill patients. Crit Care Med 2008, 12: 116.

21. Preiser JC: Restoring normoglycemia: not so harmless. Crit Care 2008, 12:116.

22. Preiser JC, Devos P: Steps for the implementation and validation of tight glucose control. Intensive Care Med 2007, 33:570571.

23. Krinsley J, Zheng $P$, Hall D, Magarian P: ICU validation of the OptiScanner, a continuous glucose monitoring device [abstract]. Crit Care Med 2006, 34:A67.

24. Bochicchio GV, Bochicchio KM, Lettich K, Lambert P, Herrera A, Lumpkins K, Scalea T, Magarian P: Cutting edge technology in tight glycemic control (TGC) [abstract]. Crit Care Med 2007, 35:A495.

25. Yamashita K, Yokoyama T, Yatabe T, Manabe M, Okabayashi T, Maeda $\mathrm{H}$, Hanazaki K: Accuracy and reliability of a continuous blood glucose monitor in critically ill patients [abstract]. Crit Care Med 2007, 35:A495. 